ISSN 2179-6750

\title{
Perfil clínico-epidemiológico e prevalência de coinfecção pelo vírus da imunodeficiência humana em pacientes com tuberculose no estado de Pernambuco
}

Recebido: 28 maio 2018

Revisado: 9 nov 2018

Aceito: $27 \mathrm{dez} 2020$

Autor de correspondência:

Eliane Maria Medeiros Leal

eliane.medeiros.leal@gmail.com

Como citar: Gonçalves HF, Leal EMM, Silva CCAV, Silva FS.

Perfil clínico-epidemiológico e prevalência de coinfecção pelo vírus da imunodeficiência humana em pacientes com tuberculose no estado de Pernambuco. J Manag Prim Health Care. 2021;13:e02.

https://doi.org/10.14295/jmphc .v13.575.

Conflito de interesses: Os autores declaram não haver nenhum interesse profissional ou pessoal que possa gerar conflito de interesses em relação a este manuscrito.

Copyright: Este é um artigo de acesso aberto, distribuído sob os termos da Licença Creative Commons (CC-BY), Esta licença permite que outros distribuam, remixem, adaptem e criem a partir do seu trabalho, mesmo para fins comerciais, desde que Ihe atribuam o devido crédito pela criação original.

\author{
Perfil clínico-epidemiológico y prevalencia de coinfección por el virus de la \\ inmunodeficiencia humana en pacientes con tuberculosis \\ del estado de Pernambuco \\ Clinical-epidemiological profile and prevalence of coinfection by human \\ immunodeficiency viruses in patients with tuberculosis \\ in the state of Pernambuco
}

Hayana Figueiredo GONÇALVES ${ }^{(1)}$

Eliane Maria Medeiros LEAL ${ }^{(2)}$

Carla Carolina Alexandrino Vicente da SILVA ${ }^{(2)}$

Filipe Santana da SILVA ${ }^{(3)}$

\begin{abstract}
(1) Especialista em Auditoria em Saúde - UNINTER, Recife, PE, Brasil.
(2) Fundação Oswaldo Cruz - Fiocruz, Instituto Aggeu Magalhães, Programa de Pós Graduação em Saúde Pública, Recife, PE, Brasil.

(3) Universidade Federal de Ciências da Saúde de Porto Alegre - UFCSPA, Departamento de Ciências Exatas e Sociais Aplicadas - DECESA, Porto Alegre, RS, Brasil.
\end{abstract}

\begin{abstract}
Resumo
A tuberculose se caracteriza como um grande desafio para a saúde pública do Brasil. Quando em concomitância com vírus da imunodeficiência humana é uma das principais causas de evolução rápida para óbito dos pacientes. Identificar o perfil clínico e epidemiológico e a prevalência de coinfecção tuberculose/ Vírus da Imunodeficiência Humana - VIH no Estado de Pernambuco. Trata-se de um estudo transversal descritivo, com abordagem quantitativa, realizado no Estado de Pernambuco, com 2.824 casos de coinfecção pelo vírus da imunodeficiência humana e pela tuberculose, através das fichas notificação de tuberculose do Sistema de Informação de Agravos e Notificação do Ministério da Saúde. Observou-se que $88 \%$ residiam na zona urbana e $68,5 \%$ eram do sexo masculino. Predominou-se a faixa etária de 20 a 40 anos (59,1\%). Dentre os exames realizados, $28,9 \%$ dos casos apresentaram resultados positivos no exame de baciloscopia de escarro na $1^{\mathrm{a}}$ amostra e na $2^{\mathrm{a}}$ amostra (11,9\%). A cultura de escarro não foi realizada em $85,5 \%$ dos casos. A coinfecção de tuberculose e VIH é um problema relevante em Pernambuco. O perfil predominante dos casos (homens, cor parda em idade economicamente ativa e na zona urbana) denota uma rapidez de ações focais de prevenção para esse grupo prioritariamente. Apesar dos programas de saúde pública existente e da antiguidade das doenças necessita-se de ações de controle de ambas.
\end{abstract}

Descritores: Coinfecção; Tuberculose; Síndrome da Imunodeficiência Adquirida. 


\title{
Resumen
}

La tuberculosis se caracteriza por ser un gran desafío para la salud pública en Brasil. Cuando está en concomitancia con el virus de la inmunodeficiencia humana, es una de las principales causas de rápida evolución hasta la muerte de los pacientes. Identificar el perfil clínico-epidemiológico y la prevalencia de coinfección tuberculosis / VIH en el estado de Pernambuco. Se trata de un estudio descriptivo transversal, con enfoque cuantitativo, realizado en el Estado de Pernambuco, con 2.824 casos de coinfección por el virus de la inmunodeficiencia humana y tuberculosis, a través de los formularios de notificación de tuberculosis del Sistema de Información Sanitaria y Notificación del Ministerio de Salud. Salud Se observó que el $88 \%$ vivía en el área urbana y el $68,5 \%$ eran hombres. Predominó el grupo de edad de 20 a 40 años (59,1\%). Entre las pruebas realizadas, el 28,9\% de los casos presentó resultado positivo en el examen de microscopía de esputo en la $1^{\mathrm{a}}$ muestra y en la $2^{\mathrm{a}}$ muestra $(11,9 \%)$. No se realizó cultivo de esputo en el $85,5 \%$ de los casos. La coinfección de tuberculosis y VIH es un problema relevante en Pernambuco. El perfil predominante de casos (hombres, morenos en edad económicamente activa y en el área urbana) muestra una rapidez de acciones preventivas focales para este grupo como una prioridad. Apesar de los programas de salud pública existentes y la antigüedad de las enfermedades, se necesitan acciones para controlar ambos.

Palabras-claves: Coinfección; Tuberculosis; Síndrome de Inmunodeficiencia Adquirida.

\begin{abstract}
Tuberculosis is characterized as a major challenge for public health in Brazil. When in concomitance with human immunodeficiency virus, it is one of the main causes of rapid evolution to the death of patients. To identify the clinical and epidemiological profile and the prevalence of tuberculosis / HIV co-infection in the State of Pernambuco. This is a descriptive cross-sectional study, with a quantitative approach, carried out in the State of Pernambuco, with 2.824 cases of co-infection by the human immunodeficiency virus and tuberculosis, through the tuberculosis notification forms of the Health Information System and Notification of the Ministry of Health. Health. It was observed that $88 \%$ lived in the urban area and $68.5 \%$ were male. The age group from 20 to 40 years old predominated (59.1\%). Among the tests performed, $28.9 \%$ of the cases presented positive results in the sputum smear microscopy exam in the 1 st sample and in the 2 nd sample (11.9\%). Sputum culture was not performed in $85.5 \%$ of cases. Coinfection of tuberculosis and HIV is a relevant problem in Pernambuco. The predominant profile of cases (men, brown in economically active age and in the urban area) shows a rapidity of focal preventive actions for this group as a priority. Despite the existing public health programs and the age of the diseases, actions are needed to control both.

Keywords: Coinfection; Tuberculosis; Acquired Immunodeficiency Syndrome.
\end{abstract}

\section{Introdução}

Tuberculose (TB), doença tão antiga, que tem tratamento eficaz e efetivo há anos, mas ainda continua sendo um grave problema de saúde pública para o Brasil. ${ }^{1}$ A TB é uma doença infectocontagiosa, ocasionada pelo Mycobacterium tuberculosis, que afeta, prioritariamente, as funções fisiológicas do sistema respiratório.

Apesar de tratável e curável, a mortalidade por essa doença ainda é bem elevada nas capitais do Brasil em que há maiores desníveis em desigualdade social. Fatores que podem estar associados à sua maior ocorrência são (por exemplo) indivíduos de segmentos sociais menos favorecidos, migrantes e pessoas com imunodeficiência, tal como os pacientes infectados pelo Vírus da Imunodeficiência Humana - VIH. ${ }^{2}$

O VIH atua sobre o sistema imunológico, responsável pela proteção do homem contra vírus, bactérias e outros microrganismos. Sendo assim, quando um indivíduo está 
infectado pelo VIH, este se torna mais suscetível a outras doenças oportunistas. Uma doença frequentemente associada à infecção pelo VIH é a TB. ${ }^{3}$

Durante o diagnóstico de TB, é frequente a descoberta de soropositividade para o VIH. No Brasil, cerca de $15 \%$ dos indivíduos diagnosticados com TB apresentam positividade para o VIH. Nesses casos, a TB é a principal causa de morte, chegando a taxas de óbito de $20 \%$ tanto pela sua evolução quanto pelos fatores associados ao abandono do tratamento. Esse índice de mortalidade pode ser agravado por dois fatores, sendo eles o aparecimento de reações adversas ao tratamento e a descoberta tardia da coinfecção. ${ }^{4}$

No que diz respeito à apresentação clínica/gravidade da TB na coinfecção TB-VIH, estudo ${ }^{5}$ descreve que pode haver variação na apresentação clínica da TB em indivíduos coinfectados, estando associado o grau de supressão imunológica do indivíduo. Todavia e independente da infecção pelo vírus VIH. Este é um dos motivos pelo qual é tão importante realizar o diagnóstico precoce da coinfecção.

Segundo a Organização Mundial de Saúde - OMS, no ano de 2015, 10,4 milhões de pessoas adoeceram com TB e 1,2 milhões de pessoas conviveriam com a coinfecção TB-VIH. Em 2014, 1,5 milhões de pessoas morreram de TB e 400 mil morreram com a coinfecção TBVIH. ${ }^{6}$ Dentre os países com maiores indicadores, o Brasil é um dos países prioritários para a intensificação de cuidados em TB. O país ocupa $20^{a}$ posição quanto à carga da doença e a $19^{\mathrm{a}}$ no que se refere à coinfecção TB-VIH.

No Brasil, houve uma redução do coeficiente de incidência de TB, frequentemente associado ao avanço da Estratégia de Saúde da Família - ESF e pelo Tratamento Diretamente Observado - TOD. Em 2001, as taxas de incidência eram de 42,7 e decaíram para 34,2 casos por 100 mil habitantes em 2014. Todavia, a TB ainda persiste como grande problema de saúde no Brasil. Em 2015, houve mais de 69 mil indivíduos acometidos pela de TB, dentre os quais 6,8 mil pessoas conviviam com a coinfecção TB-VIH. ${ }^{7}$

O "Plano Nacional pelo Fim da Tuberculose", lançado em 2017, propõe estratégias para eliminar a TB no Brasil. Dentre outras ações, é proposto a realização do diagnóstico precoce para todas as formas de TB (pulmonar ou não), com oferta universal de cultura e teste de sensibilidade, incluindo o uso de testes rápidos moleculares. Dessa forma será possível agir ainda sobre o TB-VIH, especificamente como a oferta de testes de VIH para todas as pessoas com TB. ${ }^{7}$

Mesmo nesse cenário, um dos principais agravantes da coinfecção VIH/TB é a taxa de mortalidade, que chegam a ser 19 vezes mais altas do que entre os indivíduos que não tem a coinfecção. ${ }^{8}$ O Brasil, em especial os estados da Região Nordeste, apresenta condições propícias para manutenção dos elevados índices de prevalência da infecção pelo VIH, 
associando grandes contingentes populacionais à pobreza e à desestruturação dos serviços públicos. $^{9}$

Além desses fatores associados à mortalidade, ainda há um alto índice de prevalência de morbimortalidade de TB, tais como: ${ }^{10}$

1. A modificação do caráter da doença, a qual ela passa de crônica para aguda;

2. A dificuldade de detecção precoce de VIH em pacientes com Tuberculose (conforme preconiza o Ministério da Saúde);

3. A dificuldade de adesão ao tratamento;

4. O tempo de terapêutica;

5. A resistência às drogas disponíveis e aos efeitos adversos; e

6. A própria morbidade da coinfecção TB-VIH/AIDS.

No Brasil, Pernambuco é um dos Estados com o maior número de casos de TB. ${ }^{11}$ Segundo dados de mortalidade até outubro de 2016, os Estados do Rio de Janeiro e Pernambuco se destacaram como os de maiores do coeficiente de mortalidade, 5,0 e 4,5 óbitos por TB para cada 100 mil habitantes, respectivamente. ${ }^{7}$ Nos últimos anos, a despeito da implementação de políticas e estratégias de controle, como TDO, a taxa de abandono em Pernambuco vem se mantendo em torno dos $8,3 \% .^{12}$

Considerando o contexto mundial, nacional e (especificamente) a manutenção de altos níveis de óbito e abandono do tratamento no Estado de Pernambuco para a coinfecção TB-VIH, surge a necessidade de intervenções específicas para o perfil de população mais vulnerável a essa condição. O objetivo desse estudo é identificar o perfil clínico e epidemiológico e a prevalência de coinfecção tuberculose/VIH no Estado de Pernambuco.

\section{Metodologia}

Trata-se de um estudo descritivo, retrospectivo, transversal, com abordagem quantitativa, realizado a partir do Sistema de Informação de Agravos e Notificação (SINAN) do Ministério da Saúde - MS do Brasil. Foram adotados os seguintes critérios de inclusão para participação no estudo: casos confirmados e notificados de tuberculose com coinfecção pelo VIH durante o período de 2012 a 2016. A população do estudo foi composta de 2.824 casos de coinfecção de TB-VIH, em Pernambuco.

O Estado de Pernambuco é localizado no Nordeste brasileiro e apresenta área (aproximada) de 98 milhões de quilômetros quadrados. Em 2010, a população era composta de 9.410.336 habitantes. Pernambuco apresenta um índice de desenvolvimento humano (IDH) municipal médio de 0,67 , sendo descrito como um nível 
médio de desenvolvimento humano com desigualdades sociais importantes. ${ }^{13}$ As variáveis estudadas foram:

1. Socioeconômicas e demográficas: sexo, raça/cor, faixa etária, escolaridade, zona de residência; e

2. Clínico-epidemiológica: tipo de entrada, forma clínica, baciloscopia de escarro, $1^{a}$ e $2^{a}$ amostra, cultura de escarro, TDO, situação de encerramento/desfecho do tratamento.

A coleta de dados se deu em janeiro de 2017, através da interface de tabulação do Tabnet alimentada pela Secretária Estadual de Saúde de Pernambuco. A análise dos dados se deu por meio de estatística descritiva com confecção de tabelas para a representação dos dados.

Tendo em vista que os dados utilizados foram de origem secundária e de domínio público, caracterizando a não obrigatoriedade do parecer do comitê de ética.

\section{Resultados}

Observa-se na Tabela 1 que 88,0\% dos coinfectados residiam na zona urbana e era do sexo masculino (68,5\%). Foram predominantes também a faixa etária entre 20-40 anos $(59,1 \%)$, a raça/cor parda (55,6\%), e nível educacional de $5^{a}$ a $8^{a}$ série incompleta do ensino fundamental (13,8\%).

Tabela 1. Caracterização socioeconômica e demográfica dos casos de coinfecção por VIH em pacientes com tuberculose no Estado de Pernambuco durante o período de 2012-2016

\begin{tabular}{c|c|c}
\hline \multicolumn{1}{c|}{ Variáveis } & $\mathrm{n}^{*}$ & $\%$ \\
\hline Sexo & 887 & 31,4 \\
\hline Feminino & 1.935 & 68,5 \\
\hline Masculino & 02 & 0,1 \\
\hline Ignorado & 507 & 18,0 \\
\hline Raça/Cor & 331 & 11,7 \\
\hline Branca & 21 & 0,7 \\
\hline Preta & 1.569 & 55,6 \\
\hline Amarela & 18 & 0,6 \\
\hline Parda & 378 & 13,4 \\
\hline Indígena & & \\
\hline Ignorado & 125 & 4,4 \\
\hline Faixa etária (anos) & 1.670 & 59,1 \\
\hline$<20$ & 908 & 32,2 \\
\hline $20-40$ & 121 & 4,3 \\
\hline $41-60$ & & \\
\hline$>60$ & & \\
\hline \hline
\end{tabular}


ISSN 2179-6750

\begin{tabular}{|c|c|c|}
\hline Variáveis & $n^{*}$ & $\%$ \\
\hline \multicolumn{3}{|l|}{ Escolaridade } \\
\hline Analfabeto & 309 & 10,9 \\
\hline $1^{\mathrm{a}}-4^{\mathrm{a}}$ série incompleta do $\mathrm{EF}$ & 328 & 11,6 \\
\hline $1^{\mathrm{a}}-4^{\mathrm{a}}$ série completa do EF & 163 & 5,8 \\
\hline $5^{a}-8^{a}$ série incompleta do $E F$ & 391 & 13,8 \\
\hline EF completo & 135 & 4,8 \\
\hline EM incompleto & 121 & 4,3 \\
\hline EM completo & 254 & 9,0 \\
\hline ES incompleto & 24 & 0,8 \\
\hline ES completo & 44 & 1,6 \\
\hline Não se aplica & 44 & 1,6 \\
\hline Ignorado & 1011 & 35,8 \\
\hline \multicolumn{3}{|l|}{ Zona de residência } \\
\hline Rural & 131 & 4,6 \\
\hline Urbana & 2.484 & 88,0 \\
\hline Periurbana & 13 & 0,5 \\
\hline Ignorado & 196 & 6,9 \\
\hline
\end{tabular}

Fonte: Elaborado pelos autores (2020).

Notas: Dados extraídos de TABNET/DATASUS, Secretaria Estadual de Saúde de Pernambuco. ${ }^{*} \mathrm{n}=2.824$

O presente estudo identificou que a maioria dos casos era do sexo masculino, o que condiz com outros estudos., ${ }^{9,14,15} \mathrm{Na}$ literatura não há clareza dos condicionantes da coinfecção de TB-VIH acometer mais homens. Porém, este fato pode estar associado a fatores biológicos, estilo de vida, falta de autocuidado, além de que (historicamente) os homens tendem a buscar tardiamente o serviço de saúde. ${ }^{16}$

A predominância da cor preta/parda é associada ao fato dessa população ser extremamente vulnerável do ponto de vista social, incluindo limitações de cuidados e acesso às ações e serviços de saúde. ${ }^{17}$ A maioria da população estudada é da cor parda $(55,6 \%)$ ou preta. parda e a preta $(11,7 \%)$, juntas acometeram $67,3 \%$ dos coinfectados, ou seja, a maioria.

Neste estudo, a faixa etária com maior ocorrência foi de 20 a 40 anos (59,1\%), indicando uma população de adultos jovens. Na região do Maranhão, 64\% dos casos representavam a faixa etária de 20 a 40 anos. E em Porto Alegre (Rio Grande do Sul), 83\% dos casos eram de indivíduos apresentando idade entre 20 e 59 anos. A faixa etária é considerada como uma população economicamente ativa. Esse fato pode estar relacionado ao estilo de vida de adultos e jovens, com hábitos vulneráveis (como o não uso de métodos contraceptivos de barreira, tal como a camisinha) e uso de drogas ilícitas. ${ }^{3,14}$

A baixa escolaridade é um fator importante, que pode influenciar diretamente na grande quantidade de casos de TB-VIH. Historicamente a escolaridade impacta diretamente nas condições de saúde do indivíduo devido à dificuldade de obtenção de informações 
acerca do acesso aos serviços de saúde e ao melhor entendimento da doença e de seus determinantes e condicionantes. ${ }^{3}$

Nesse sentido, é associada a pessoas com baixa escolaridade a pobreza e ao menor acesso a informações. Ou seja, pode existir um menor entendimento dos riscos que elas estão expostas. ${ }^{18}$ No que se referem ao contexto sociopolítico, esses mesmos indivíduos acabam se envolvendo em violência, tráfico e uso de drogas, prostituição, alcoolismo entre outros $^{19}$ que podem levar a comportamentos de risco possibilitando maior exposição ao VIH, por exemplo. Esse contexto aponta que a TB e a Síndrome da Imunodeficiência Adquirida (SIDA, mais conhecida pelo acrônimo em inglês como AIDS) são doenças que ultrapassam as barreiras biológicas e chegam a ser um problema social. ${ }^{16}$

Observa-se que a prevalência de coinfecção em Pernambuco é mais alta na zona urbana com $88 \%$ dos casos, isso pode ser explicado pelo fato da população urbana ser maior que a da zona rural. A urbanização desenfreada e desorganizada, a pobreza e má distribuição de renda trouxeram maior contato com condições vulneráveis de doenças como a AIDS e a TB. ${ }^{20}$

A taxa de coinfecção de TB-VIH no Brasil gira em torno dos $10 \%$, sendo a Região Sul com a maior porcentagem (18,2\%). Já a Região Nordeste encontra-se abaixo da média do Brasil, com um percentual de 8,5. Pernambuco é o Estado que tem o maior número de casos de coinfecção de TB-VIH, com 12\%. Dessa forma, Pernambuco encontra-se acima da média do Brasil e região. ${ }^{21}$ A caracterização clínico-epidemiológica desses casos encontra-se descrita na Tabela 2.

Tabela 2. Caracterização clínico-epidemiológica dos casos de coinfecção pelo VIH em pacientes com tuberculose no Estado de Pernambuco durante o período de 2012-2016

\begin{tabular}{c|c|c}
\hline \multicolumn{1}{c|}{ Variáveis } & $\mathrm{n}^{\star}$ & $\%$ \\
\hline Tipo de entrada & 1.967 & 69,60 \\
\hline Caso novo & 175 & 6,21 \\
\hline Recidiva & 514 & 18,21 \\
\hline Reingresso após abandono & 135 & 4,78 \\
\hline Transferência & 23 & 0,82 \\
\hline Não sabe & 10 & 0,35 \\
\hline Pós-óbito & & \\
\hline Forma clínica & 1.964 & 69,50 \\
\hline Pulmonar & 544 & 19,3 \\
\hline Extrapulmonar & 316 & 11,2 \\
\hline Pulmonar + Extrapulmonar & & \\
\hline Baciloscopia de escarro (1 ${ }^{\text {a }}$ amostra) & 810 & 28,90 \\
\hline Positivo & 629 & 22,20 \\
\hline Negativo & & \\
\hline \hline
\end{tabular}


ISSN 2179-6750

\begin{tabular}{|c|c|c|}
\hline Variáveis & $n^{*}$ & $\%$ \\
\hline Não realizada & 1.359 & 48,00 \\
\hline Não informada & 26 & 0,90 \\
\hline \multicolumn{3}{|l|}{ Baciloscopia de escarro ( $2^{\mathrm{a}}$ amostra) } \\
\hline Positivo & 337 & 11,70 \\
\hline Negativo & 367 & 13,00 \\
\hline Não realizada & 1.079 & 38,60 \\
\hline Não informada & 1.041 & 36,80 \\
\hline \multicolumn{3}{|l|}{ Cultura de escarro } \\
\hline Positiva & 132 & 4,70 \\
\hline Negativa & 92 & 3,30 \\
\hline Em andamento & 185 & 6,50 \\
\hline Não realizada & 2.415 & 85,50 \\
\hline \multicolumn{3}{|l|}{ Tratamento supervisionado (TDO) } \\
\hline Sim & 684 & 24,20 \\
\hline Não & 1.347 & 47,70 \\
\hline Ignorado/Não informado & 793 & 28,10 \\
\hline \multicolumn{3}{|l|}{ Encerramento do caso } \\
\hline Cura & 820 & 29,04 \\
\hline Abandono & 538 & 19,05 \\
\hline Óbito por tuberculose & 45 & 1,59 \\
\hline Óbito por outras causas & 528 & 18,67 \\
\hline Transferência & 478 & 16,96 \\
\hline TB resistente aos medicamentos (TB-DR) & 12 & 0,42 \\
\hline Mudança de esquema & 12 & 0,42 \\
\hline Ignorado/Branco & 391 & 13,85 \\
\hline
\end{tabular}

Fonte: Elaborado pelos autores (2020).

Notas: Dados extraídos de TABNET/DATASUS. Secretaria Estadual de Saúde de Pernambuco. *n=2.824.

Observou-se que a forma clínica predominante foi a TBC pulmonar (69,5\%), sendo 69,65\% de casos novos. Em relação aos exames, 28,9\% dos casos apresentaram resultados positivos na baciloscopia de escarro na $1^{a}$ amostra; e 11,9\% na $2^{a}$ amostra. A cultura de escarro não foi realizada em $85,5 \%$ dos casos e o resultado foi positivo para $4,7 \%$. Observa-se ainda que $47,7 \%$ não fizeram tratamento supervisionado. Quanto ao encerramento dos casos, a maior parte evoluiu para a cura (29,04\%). Os casos em retratamento são mais facilmente submetidos à realização da cultura de escarro, e com resultado positivo na baciloscopia de escarro, tanto na primeira quanto na segunda amostra numa proporção maior. ${ }^{11}$

O elevado número de casos novos $(69,6 \%)$ em Pernambuco caracteriza ainda a grande incidência da doença no Estado. Em outro estudo, o percentual encontrado para uma região do Maranhão foi de $73 \%{ }^{3}$ Esse fato pode estar associado a uma melhora no diagnóstico, ou a maior busca para realização do exame, ou (ainda) falhas na prevenção da TB. Isso pode vir a gerar um aumento de casos de coinfecção como VIH e vice-versa. 
O diagnóstico precoce da TB é importante para que seja dado início ao tratamento, evitando a evolução da doença. Nos casos de TB-VIH, o diagnóstico precoce dispõe a melhora do quadro clínico e maior possibilidade de cura. A baciloscopia é fundamental na coinfecção para confirmar a presença da TB ativa, por ter baixo custo e ser um método rápido. ${ }^{11}$ Mas, em casos cujo sujeito seja suspeito de TB através do diagnóstico por radiologia com a baciloscopia negativa opta-se pela realização da cultura.

Todavia, em casos cujo indivíduo seja suspeito de forma radiológica de TB pulmonar com a baciloscopia negativa, opta-se pela realização da cultura. ${ }^{3}$ O presente estudo evidencia que foi realizada a baciloscopia de $1^{\text {a }}$ amostra em 50,9\% dos casos. Porém, a porcentagem da não realização da baciloscopia foi alta, com 48,1\%. Já na $2^{a}$ amostra, a porcentagem de baciloscopia não realizada $(38,7 \%)$ foi maior do que as realizadas $(24,9 \%)$.

Em ternos de apresentação clínica, a predominância de TB pulmonar na coinfecção observada nesse estudo (69,5\%) é corroborada por outros. ${ }^{3,8,9,15}$ No entanto os quase $20 \%$ de casos extrapulmonar, podem estar relacionados às características de baixa imunidade dos portadores do vírus VIH. ${ }^{15}$

O TDO deve ser realizado em todos os pacientes de tuberculose como é preconizado pelo Ministério da Saúde. O estudo realizado na Região de Tocantins/MA mostra a prevalência da supervisão do tratamento, diferentemente dos resultados encontrados neste estudo, em que aproximadamente $50 \%$ não realizam o TODO. ${ }^{3} \mathrm{~A}$ realização do TDO é importante para que o medicamento seja tomado corretamente, pois o tratamento da tuberculose é realizado por meio de antibióticos com alta de probabilidade de cura se administrado corretamente. Considerando que o tratamento tem duração de 6 meses, a longa duração pode levar ao abando do tratamento.

O Ministério da Saúde - MS define uma média nacional de que 85\% dos casos de tuberculose sejam curados com uma taxa de $5 \%$ de abandono do tratamento. O presente estudo evidencia cura em $24,04 \%$ dos casos, abaixo do que é proposto pelo MS. Esse indicador é inferior ao que foi identificado em estudos semelhantes $(39,03 \%)^{9}$ e (62\%). ${ }^{3}$ Em estudo realizado em Fortaleza/Ceará evidenciou-se uma baixa taxa de cura e alta taxa de abandono também, caracterizando talvez uma tendência nacional. ${ }^{15}$ No presente estudo, a taxa de abandono foi cerca de 4 vezes maior que a preconizada pelo MS. Esse resultado sugere que serviços de saúde municipais e estaduais devem se aperfeiçoar no acompanhamento e monitoramento de pacientes com coinfecção TB-VIH, no intuito de ampliar cura e diminuir taxas de abandono. 


\section{Conclusão}

Em Pernambuco, o número de casos de coinfecção TB-VIH é um problema iminente de saúde pública. O perfil predominante dos casos (homens, cor parda em idade economicamente ativa e na zona urbana) denota uma rapidez de ações focais de prevenção para esse grupo prioritariamente.

Diante dos programas de saúde pública existentes, ainda há muito que ser feito. Os principais pontos de atuação de acordo com o perfil descrito nesse estudo podem ser:

1. A necessidade de realização de teste de VIH para pessoas com tuberculose;

2. A intensificação de ações de prevenção nos grandes centros urbanos, principalmente ao público masculino adulto jovem;

3. A facilitação e a viabilização do acesso ao diagnóstico precoce e ao TDO na atenção básica; e

4. A intensificação de ações de educação permanente para os profissionais de saúde a fim de uma maior sensibilização e melhor atuação nos diversos níveis de atendimento em saúde para as doenças.

A necessidade de estudos que permitam descrever o perfil dos pacientes e a prevalência da coinfecção TB-VIH é evidente. Este estudo permitiu conhecer a situação clínica e epidemiológica dessa população a fim de subsidiar ações mais especificas e efetivas para o controle da coinfecção TB-VIH. Além disso, a coinfecção tuberculose/VIH se destaca como um importante indicador da qualidade dos serviços de saúde. Isso permite abrir possibilidades para reflexões sobre as práticas em saúde, em particular de Pernambuco, comparar com a realidade de outras regiões do Brasil e do mundo.

Mesmo apresentando indicadores relevantes, podemos citar algumas limitações no nosso estudo. É possível citar a questão das dificuldades dos serviços em alimentar o sistema de informação com a completude dos dados. Muitos dados não informados. Isso pode estar relacionado ao mau preenchimento das fichas de notificação pelos profissionais de saúde, ou erros no momento da digitação do sistema on line.

Isso pode gerar dados errôneos e consequentemente informações aquém ou além da verdade. Esse panorama pode ser indicação de necessidade de investimentos em educação continuada para os atores envolvidos no processo, visando à maior garantia de fidelidade e confiabilidade das informações dos sistemas informatizados no Brasil e consequentemente da descrição e análise epidemiológica.

\section{Contribuição autoral}

H. F. Gonçalves realizou a escrita, a análise, interpretação dos dados dos dados. E. M. M. 
Leal realizou a escrita, a análise, interpretação dos dados dos dados e revisou o texto completo. C. C. A. V. Silva realizou interpretação dos dados e revisou o texto completo. F. S. Silva realizou escrita, avaliação dos dados, revisão do texto e aprovação do formato final.

\section{Referências}

1. Hijjar MA, Procópio MJ. Tuberculose: epidemiologia e controle no Brasil. Rev HUPE [Internet]. 2006 [citado 3 mar. 2021];5(2):15-23. Disponível em:

http://revista.hupe.uerj.br/detalhe_artigo.asp?id=228

2. Ceccon RF, Maffacciolli R, Burille A, Meneghel SN, Oliveira DLLC, Gerhardt TE. Mortalidade por tuberculose nas capitais brasileiras, 2008-2010. Epidemiol Serv Saude. 2017;26(2):34958. https://doi.org/10.5123/s1679-49742017000200012.

3. Santos Neto M, Silva FL, Sousa KR, Yamamura M, Popolin MP, Arcêncio RA. Perfil clínico e epidemiológico e prevalência da coinfecção tuberculose/VIH em uma regional de saúde no Maranhão. J Bras Pneumol. 2012;38(6):724-32. https://doi.org/10.1590/S180637132012000600007.

4. Ministério da Saúde (BR), Secretaria de Vigilância em Saúde, Departamento de Vigilância Epidemiológica. Manual de recomendações para o controle da tuberculose no Brasil [Internet].2a ed. atual. Brasília, DF: MS; 2011 [citado 3 mar. 2021]. 284 p. Disponível em:

http://bvsms.saude.gov.br/bvs/publicacoes/manual_recomendacoes_controle_tuberculose_brasil.pdf

5. Barbosa IR, Costa ICC. A emergência da co-infecção tuberculose-VIH no Brasil. Hygeia [Internet]. 2012 [citado 3 mar. 2021];8(15):232-44. Disponível em: http://www.seer.ufu.br/index.php/hygeia/article/view/17350/11177

6. World Health Organization. Global tuberculosis report 2016 [Internet]. Geneva: WHO; 2016 [citado 3 mar. 2021]. 201 p. Disponível em: http://apps.who.int/medicinedocs/en/d/Js23098en/

7. Ministério da Saúde (BR), Secretaria de Vigilância em Saúde, Departamento de Vigilância das Doenças Transmissíveis. Brasil livre de tuberculose: plano nacional pelo fim da tuberculose como problema de saúde pública [Internet]. Brasília, DF: MS; 2017 [citado 3 mar. 2021]. 52 p. Disponível em: http://portalarquivos2.saude.gov.br/images/pdf/2017/junho/29/plano_nacional_tb_web.pdf

8. Carvalho LGM, Buani AZ, Zöllner MSAC, Scherma AP. Co-infecção por mycobacterium tuberculosis e vírus da imunodeficiência humana: uma análise epidemiológica em Taubaté (SP). J Bras Pneumol. 2006;32(5):424-9. https://doi.org/10.1590/S1806-37132006000500009. 
9. Barbosa IR, Costa ICC. Estudo epidemiológico da coinfecção tuberculose-VIH no nordeste do Brasil. Rev Patol Trop. 2014;43(1):27-38. https://doi.org/10.5216/rpt.v43i1.29369.

10. Piller RVB. Epidemiologia da tuberculose. Pulmao RJ [Internet]. 2012 [citado 3 mar. 2021];21(1):4-9. Disponível em: http://www.sopterj.com.br/wpcontent/themes/_sopterj_redesign_2017/_revista/2012/n_01/02.pdf

11. Silva CCAV, Andrade MS, Cardoso MD. Fatores associados ao abandono do tratamento de tuberculose em indivíduos acompanhados em unidades de saúde de referência na cidade do Recife, Estado de Pernambuco, Brasil, entre 2005 e 2010. Epidemiol Serv Saude. 2013;22(1):77-85. http://dx.doi.org/10.5123/S1679-49742013000100008.

12. Ministério da Saúde (BR), Secretaria de Vigilância em Saúde. Indicadores prioritários para o monitoramento do Plano Nacional pelo Fim da Tuberculose como problema de saúde pública no Brasil. Bol Epidemiol [Internet]. 2017 [citado 3 mar. 2021];48(8):1-11. Disponível em: http://portalarquivos2.saude.gov.br/images/pdf/2017/marco/23/2017-V-48-N-8Indicadores-priorit--rios-para-o-monitoramento-do-Plano-Nacional-pelo-Fim-daTuberculose-como-Problema-de-Sa--de-P--blica-no-Brasil.pdf

13. Instituto Brasileiro de Geografia e Estatística. Características da população e dos domicílios: resultados do universo. Rio de Janeiro: IBGE; 2011.

14. Peruhype RC, Acosta LMW, Ruffino-Netto A, Oliveira MMC, Palha PF. The distribution of tuberculosis in Porto Alegre: analysis of the magnitude and tuberculosis-VIH coinfection. Rev Esc Enferm USP. 2014;48(6):1035-43. https://doi.org/10.1590/S0080623420140000700011.

15. Pires Neto RJ, Gadelha RRM, Herzer TL, Peres DA, Leitão TMJS, Façanha MC, et al. Características clínico-epidemiológicas de pacientes com coinfecção VIH/tuberculose acompanhados nos serviços de referência para VIH/AIDS em Fortaleza, Ceará, entre 2004 e 2008. Cad Saude Colet [Internet]. 2012 [citado 3 mar. 2021];20(2):244-9. Disponível em: http://www.cadernos.iesc.ufrj.br/cadernos/images/csc/2012_2/artigos/csc_v20n2_244 249.pdf

16. Santos MLSG, Ponce MAZ, Vendramini SHF, Villa TCS, Santos NSGM, Wysocki AD, et al. The epidemiological dimension of TB/HIV co-infection. Rev Latino-Am Enferm. 2009;17(5):683-8. http://dx.doi.org/10.1590/S0104-11692009000500014.

17. Sonnenberg P, Glynn JR, Fielding K, Murray J, Godfrey-Faussett P, Shearer S. How soon after infection with VIH does the risk of tuberculosis start to increase?: a retrospective cohort study in South African gold miners. J Infect Dis. 2005;191(2):150-8. http://dx.doi.org/10.1086/426827. 
ISSN 2179-6750

18. Silveira JM, Mendoza Sassi RA, Oliveira Netto IC, Hetzel JL. Prevalência e fatores associados à tuberculose em pacientes soropositivos para o vírus da imunodeficiência humana em centro de referência para tratamento da síndrome da imunodeficiência adquirida na região sul do Rio Grande do Sul. J Bras Pneumol. 2006;32(1):48-55. https://doi.org/10.1590/S1806-37132006000100011.

19. Prado TN, Rajan JV, Miranda AE, Dias ES, Cosme LB, Possuelo LG, et al. Clinical and epidemiological characteristics associated with unfavorable tuberculosis treatment outcomes in TB-VIH co-infected patients in Brazil: a hierarchical polytomous analysis. Braz J Infect Dis. 2017;21(2):162-70. https://doi.org/10.1016/j.bjid.2016.11.006

20. Vendramini SHF, Gazetta CE, Chiaravalotti Netto F, Cury MR, Meirelles EB, Kuyumjian FG, et al. Tuberculose em município de porte médio do Sudeste do Brasil: indicadores de morbidade e mortalidade, de 1985 a 2003. J Bras Pneumol. 2005;31(3):237-43. https://doi.org/10.1590/S1806-37132005000300010.

21. Ministério da Saúde (BR), Secretaria de Vigilância em Saúde. Protocolo clínico e diretrizes terapêuticas para atenção integral às pessoas com infecções sexualmente transmissíveis. Brasília, DF: MS; 2015 [citado 3 mar. 2021]. Disponível em: http://bvsms.saude.gov.br/bvs/publicacoes/protocolo_clinico_diretrizes_terapeutica_atenc ao_integral_pessoas_infeccoes_sexualmente_transmissiveis.pdf

\section{Minicurrículos}

Hayana Figueiredo Gonçalves | https://orcid.org/0000-0002-6368-3473 http://lattes.cnpq.br/1169529694728742

Carla Carolina Alexandrino Vicente da Silva | https://orcid.org/0000-0002-7756-1784 http://lattes.cnpq.br/0126171269361106

Eliane Maria Medeiros Leal | https://orcid.org/0000-0002-6052-6501 http://lattes.cnpq.br/0056126015443324

Filipe Santana da Silva | http://lattes.cnpq.br/8268902147804566 http://orcid.org/0000-0002-6803-1407 